\title{
In-situ glyoxalization during biosynthesis of bacterial cellulose
}

\author{
Cristina Castro $^{\mathrm{a}, 1}$, Nereida Cordeiro $^{\mathrm{b}}$, Marisa Faria $^{\mathrm{b}}$, Robin Zuluaga ${ }^{\mathrm{a}, *}$, Jean-Luc Putaux ${ }^{\mathrm{c}, 2}$, \\ Ilari Filpponen ${ }^{\mathrm{d}}$, Lina Velez ${ }^{\mathrm{a}}$, Orlando J. Rojas ${ }^{\mathrm{d}}$, Piedad Gañán ${ }^{\mathrm{a}}$ \\ a School of Engineering, Universidad Pontificia Bolivariana, Circular 1 no. 70-01, Medellín, Colombia \\ ${ }^{\mathrm{b}}$ Competence Centre in Exact Science and Engineering, University of Madeira, 9000-390 Funchal, Portugal \\ c Centre de Recherches sur les Macromolécules Végétales (CERMAV-CNRS), Affiliated with Université Joseph, BP 53, F-38041 Grenoble Cedex 9, France. \\ ${ }^{\mathrm{d}}$ Biobased Colloids and Materials group (BiCMat), Department of Forest Products Technology, Aalto University, School of Chemical Technology, \\ P.O. Box 16300, 00076 Aalto, Espoo, Finland.
}

\section{A R T I C L E I N F O}

\section{Article history:}

Received 7 November 2014

Received in revised form 3 March 2015

Accepted 4 March 2015

Available online 14 March 2015

\section{Keywords:}

Bacterial cellulose

Gluconacetobacter medellensis

Crosslinking

Glyoxal

Surface energy

\begin{abstract}
A B S T R A C T
A novel method to synthesize highly crosslinked bacterial cellulose (BC) is reported. The glyoxalization is started in-situ, in the culture medium during biosynthesis of cellulose by Gluconacetobacter medellensis bacteria. Strong crosslinked networks were formed in the contact areas between extruded cellulose ribbons by reaction with the glyoxal precursors. The crystalline structure of cellulose was preserved while the acidic component of the surface energy was reduced. As a consequence, its predominant acidic character and the relative contribution of the dispersive component increased, endowing the BC network with a higher hydrophobicity. This route for in-situ crosslinking is expected to facilitate other modifications upon biosynthesis of cellulose ribbons by microorganisms and to engineer the strength and surface energy of their networks.
\end{abstract}

(C) 2015 Elsevier Ltd. All rights reserved.

\section{Introduction}

Bacterial cellulose (BC) is an extracellular biopolymer produced by microorganisms belonging to the Gluconacetobacter genus. These bacteria extrude cellulose ribbons that form a threedimensional network at the air-liquid medium interface. Cellulose chains are assembled into sub-elementary fibrils of $1.5 \mathrm{~nm}$ width that further associate to form cellulose nanofibrils ( $2-4 \mathrm{~nm}$ width) and nanofibril bundles or ribbons (20-100 nm width) (Iguchi, Yamanaka, \& Budhiono, 2000). BC is highly pure and crystalline and exhibit good water-holding capacity, mechanical strength and degradability (Bielecki, Krystynowicz, Turkiewicz, \& Kalinowska, 2005). As a consequence, recent investigations have considered BC as a reinforcing constituent in advanced thermoplastic and thermosetting polymer matrices, resulting in composite materials with unique properties and morphologies (Laborie, 2009).

The hydrophilicity of cellulose is related to the presence of three different $\mathrm{OH}$ groups in the repeating units of the polymer

\footnotetext{
* Corresponding author. Tel.: +57 4 4488388; fax: +57 42502080.

E-mail addresses: cristina.castro@upb.edu.co (C. Castro), robin.zuluaga@upb.edu.co (R. Zuluaga).

1 Tel.: +57 4 4488388; fax: +57 42502080 .

2 Member of Institut de Chimie Moléculaire de Grenoble and Institut Carnot PolyNat.
}

which are capable of forming inter- and intra-chain hydrogen bonds. The resultant surface hydrophilicity of cellulose fibrils limits their compatibility with nonpolar matrices. As a result, several functionalization strategies have been attempted, including esterification, polycondensation, etherification, and acetalyzation reactions (Heinze, \& Liebert, 2001). Likewise, cellulose can be chemically modified with crosslinking agents to covalently bond the fibrils (Schramm \& Rinderer, 2002; Yu, Lee, \& Bang, 2008). One of the most commonly used crosslinking agents include formaldehydebased chemicals but their use has been undermined by reported carcinogenic effects (Schramm, \& Rinderer, 2000). Thus, alternative crosslinking agents are needed such as polycarboxylic acid and dialdehydes, which have been used since the late 1980s (Xu, Yang, \& Deng, 2001; Lee, \& Kim, 2005). Among crosslinking agents, glyoxal has been one of the few readily available non-formaldehyde agents capable of crosslinking cellulose (Schramm \& Rinderer, 2002; Welch, 1983; Head, 1958). Glyoxal is the simplest of the dialdehyde group. It is produced by gas-phase oxidation of ethylene glycol, liquid-phase oxidation of acetaldehyde or by lipid autoxidation (Kielhorn, Pohlenz-Michel, Schmidt, \& Mangelsdorf, 2004). Moreover, upon release into the environment, glyoxal is rapidly converted enzymatically to glycolate by microorganisms as bacteria and fungi (Kielhorn et al., 2004; Quero et al., 2011).

Reactions with glyoxal have commonly been used to impart durable properties to cellulose-based materials, including textiles and paper (Schramm \& Rinderer, 2002; Yu et al., 2008). Surface 
modification of BC can be carried out by impregnation, primarily to reduce their hydrophilic character and prevent delamination of the nanostructure. The effects of surface modification and resulting mechanical properties have been evaluated by Quero et al. who reported a low degree of delamination while preserving the mechanical strength (Quero et al., 2011).

In the present report, we propose a novel method to glyoxalize $\mathrm{BC}$ in situ, during synthesis in the culture medium. To this end, the culture medium of Gluconacetobacter bacteria was modified with low concentration glyoxal crosslinking agent thereby allowing effective contact with the $\mathrm{BC}$ ribbons. The effects of the crosslinking reactions after thermal curing were investigated in terms of changes in morphological, chemical and crystallinity characteristics of $\mathrm{BC}$ as determined by scanning and transmission electron microscopy (SEM and TEM), X-ray diffraction (XRD), attenuated total reflection Fourier transform infrared spectroscopy (ATR-FTIR) and $\mathrm{CP} / \mathrm{MAS}{ }^{13} \mathrm{C}$ nuclear magnetic resonance (NMR). In addition, the changes in the surface properties were evaluated by inverse gas chromatography (IGC) and contact angle (CA) measurement.

\section{Materials and methods}

\subsection{Bacterial cellulose production}

The Gluconacetobacter strain used in this study was previously isolated from a pellicle of homemade vinegar (Castro et al., 2013). The purified bacterial strain was incubated at $28^{\circ} \mathrm{C}$ for 8 days in a static Hestrin-Schramm (HS) culture medium containing 2 $\mathrm{w} / \mathrm{v} \%$ glucose, $0.5 \mathrm{w} / \mathrm{v} \%$ peptone, $0.5 \mathrm{w} / \mathrm{v} \%$ yeast extract, $0.27 \mathrm{w} / \mathrm{v} \%$ $\mathrm{Na}_{2} \mathrm{HPO}_{4}$, adjusted to $\mathrm{pH} 3.5$ by phosphoric acid (also used as catalyst agent in the crosslinking reaction). Glyoxal was used as crosslinking agent and was added to the culture medium at 0.3 $\mathrm{v} / \mathrm{v} \%$. It is important to note that glyoxal concentration of $0.3 \mathrm{v} / \mathrm{v} \%$ is toxic for the microorganism. In this work, an adaptation procedure is applied. The bacteria were exposed to an initial concentration of glyoxal of $0.01 \mathrm{v} / \mathrm{v} \%$ for 8 days at $28^{\circ} \mathrm{C}$. After this time, a portion of the above medium was inoculated $(10 \mathrm{v} / \mathrm{v} \%)$ in fresh medium with a $0.02 \mathrm{v} / \mathrm{v} \%$ glyoxal concentration $(0.01 \%$ increments were made at each change). This procedure was repeated until a concentration of glyoxal of $0.3 \%$ was achieved. At each step the phenotypic characteristics of the microorganism and the ability to produce cellulose was verified using the method of Castro et al. (2013).

The $\mathrm{BC}$ pellicles collected from the final $0.3 \%$ glyoxal containing modified medium (BCM) were dried $\left(40^{\circ} \mathrm{C}, 48 \mathrm{~h}\right)$ and then cured $\left(120^{\circ} \mathrm{C}, 5 \mathrm{~min}\right)$ to allow cross-linking and to yield glyoxylated $\mathrm{BC}$ (BCG). BCG was washed with distilled water, treated for $14 \mathrm{~h}$ in a $5 \mathrm{wt} \% \mathrm{KOH}$ solution and rinsed until $\mathrm{pH} 7$ to remove the bacterial cells and residual culture medium. A control system consisting of $\mathrm{BC}$ with no glyoxal treatment $(\mathrm{BC})$ was produced following the same methodology described for BCG in glyoxal-free culture media.

\subsection{Imaging with scanning and transmission electron microscopy (SEM and TEM)}

The surface of freeze-dried pellicles was coated with gold/ palladium using an ion sputter coater for $5 \mathrm{~min}$ and the samples were imaged in a scanning electron microscope (Jeol JSM 5910 LV) using the secondary electron mode at $10 \mathrm{kV}$.

Cellulose ribbons were extracted from the BC, BCM and BCG pellicle networks by mechanical treatment at $3000 \mathrm{rpm}$ for $30 \mathrm{~s}$. Drops of each suspension were deposited onto glow-discharged carboncoated electron microscopy grids and negatively stained with $2 \mathrm{wt} \%$ uranyl acetate. All samples were observed in a transmission electron microscopy (Philips CM200) operating at $80 \mathrm{kV}$. The images were recorded on Kodak SO163 films.

\subsection{Spectroscopic analyses via FTIR and CP/MAS ${ }^{13} \mathrm{C} N M R$}

Attenuated total reflection Fourier transform infrared spectroscopy (ATR-FTIR) was used for chemical analysis. Before sample analysis, $\mathrm{BC}$ and $\mathrm{BCG}$ were dried at $40^{\circ} \mathrm{C}$ to remove moisture. FTIR spectra were recorded on a Nicolet 6700 spectrophotometer in the $4000-400 \mathrm{~cm}^{-1}$ range using ATR. The spectra were recorded with a resolution of $4 \mathrm{~cm}^{-1}$ and an accumulation of 64 scans.

${ }^{13} \mathrm{C}$ cross polarization magic angle spinning nuclear magnetic resonance (CP/MAS ${ }^{13} \mathrm{C}$ NMR) was used for analysis of the $\mathrm{BC}$ and BCG films on a Bruker AV-400-WB spectrometer with a triple probe channel of $4 \mathrm{~mm}$, with rotors of ZrO and a stopper of Kel-F at room temperature. The speed of rotation was $8 \mathrm{kHz}$ and the pulse sequence employed was cross polarization (CP-MAS) ${ }^{1} \mathrm{H}-{ }^{13} \mathrm{C}$, using a spectral width of $35 \mathrm{kHz}$, a contact time of $3 \mathrm{~ms}$ and a relaxation time of $4 \mathrm{~s}$ with decoupling ${ }^{1} \mathrm{H}$. The number of scans was 2048 . The chemical shift was established in relative ppm to tetramethylsilane (TMS) as primary reference and the signal of adamantine $\mathrm{CH}_{2}$ (29.5 ppm) was used as secondary reference.

\section{4. $X$-ray diffraction $(X R D)$}

Dry films of BC and BCG were X-rayed using a Panalytical X'Pert Pro MPD equipment operating at the Ni-filtered $\mathrm{CuK \alpha} \alpha_{1}$ radiation wavelength $(\lambda=0.15406 \mathrm{~nm})$, generated at a voltage of $45 \mathrm{kV}$ and a filament emission of $40 \mathrm{~mA}$. Data were collected in reflection mode in the $10-30^{\circ} 2 \theta$-range with a step of $0.013^{\circ}$. The scans proceeded at $56.58 \mathrm{~s}$ per step. The peaks were deconvoluted using Pearson VII peak functions for $d$-spacings and apparent crystal size (ACS) determinations. The $d$-spacings between the crystal planes were determined using Bragg's law:

$d=\frac{\lambda}{2 \sin \theta}$

where $\theta$ is the angle between the plane and the diffracted or incident beam and $\lambda$ is the X-rays wavelength. The ACS was calculated using Scherrer's formula:

$\mathrm{ACS}=\frac{(0.9 \lambda)}{\mathrm{FWHM} \cos \theta}$

where FWHM is the width of the peak at half the maximum height.

\subsection{Surface energy via contact angle (CA) measurement and inverse gas chromatography (IGC)}

Six sessile contact angles were measured on BC and BCG films using an optical contact angle meter (KSV instruments LTD) at room temperature after deposition of $5 \mu \mathrm{L}$ ultra pure water drop on the surface.

IGC measurements were carried out using a commercial inverse gas chromatograph (Surface Measurements Systems, London, UK) equipped with flame ionization (FID) and thermal conductivity (TCD) detectors. The data obtained were analyzed in iGC Standard v1.3 and Advanced Analysis Software v1.21 (Cordeiro, Gouveia, Moraes, \& Amico, 2011). The BC and BCG samples were packed into standard glass silanized columns (ID: $2 \mathrm{~mm}$, length: $30 \mathrm{~cm}$ ). The columns with the samples were then conditioned in the IGC overnight at $40^{\circ} \mathrm{C}$ followed by $2 \mathrm{~h}$ at the specific measurement condition.

An alkane series ( $n$-heptane, $n$-octane, $n$-nonane and $n$-decane) was used in the measurement of the dispersive surface interactions at $20^{\circ} \mathrm{C}$. For the acid-base studies, and specific surface free energy, acetonitrile, ethyl acetate, ethanol, acetone and tetrahydrofuran were used at the same conditions than that used for dispersive surface interaction measurements. The isotherms were collected with different concentrations of $n$-octane, ethanol and tetrahydrofuran, 

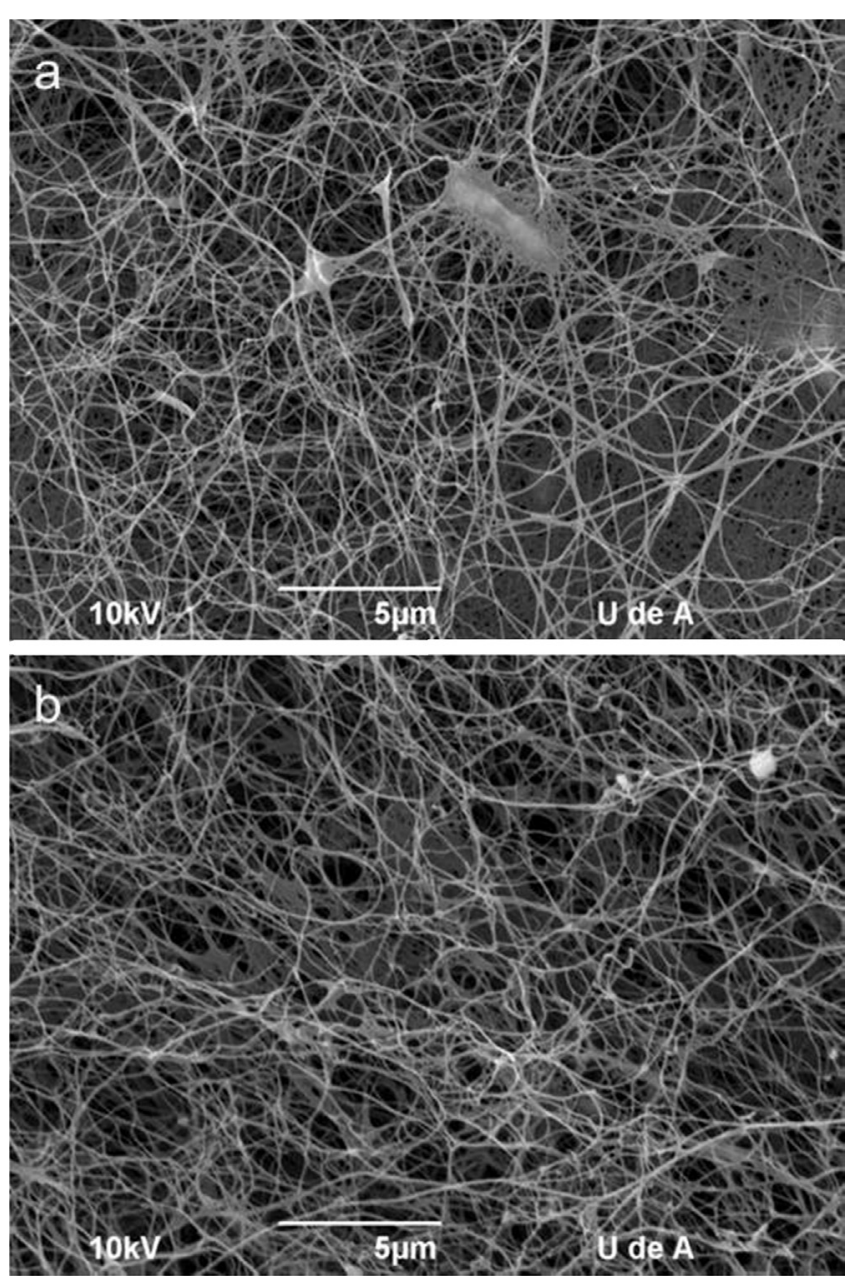

Fig. 1. SEM images of a cellulose network produced in unmodified (a) and modified media before crosslinking curing (b).

at $20^{\circ} \mathrm{C}$ and a flow rate of $10 \mathrm{~mL} \mathrm{~min}^{-1}$. All measurements of the apparent crystal size (ACS) were carried out at $0 \%$ relative humidity and methane was used as the reference molecule for calculating the dead time. The experiments were performed in duplicate and the presented results are the average values. The experimental error due to the temperature variation, flow rate and retention time measurement was estimated to be below $4 \%$.

\section{Results and discussion}

Compared with regular synthesis procedures, no changes in $B C$ production were observed after the adaptation methodology applied on the strain. Morphology of the BC in the regular and modified synthesis were observed by SEM. Fig. $1 \mathrm{a}$ and $\mathrm{b}$ shows cellulose ribbon networks formed in the medium without (BC) and with glyoxal modification (BCM), respectively. The formation of an interconnected network was not affected by the presence of glyoxal. The changes in the width of the ribbons produced in glyoxal-modified media appear to be negligible.

TEM images of BC, BCM and BCG cellulose ribbons are shown in Fig. 2a-c, respectively. The morphology of the $\mathrm{BC}$ ribbons in Fig. 2a appears not to be affected by the synthesis in the glyoxal-modified medium, BCM (Fig. 2b). However, comparatively smaller ribbons are observed after curing in BCG (Fig. 2c). This may be due to surface modification of the nanofibrils with glyoxal, which generates a compaction and therefore a decrease in the width of the ribbons during heat curing.
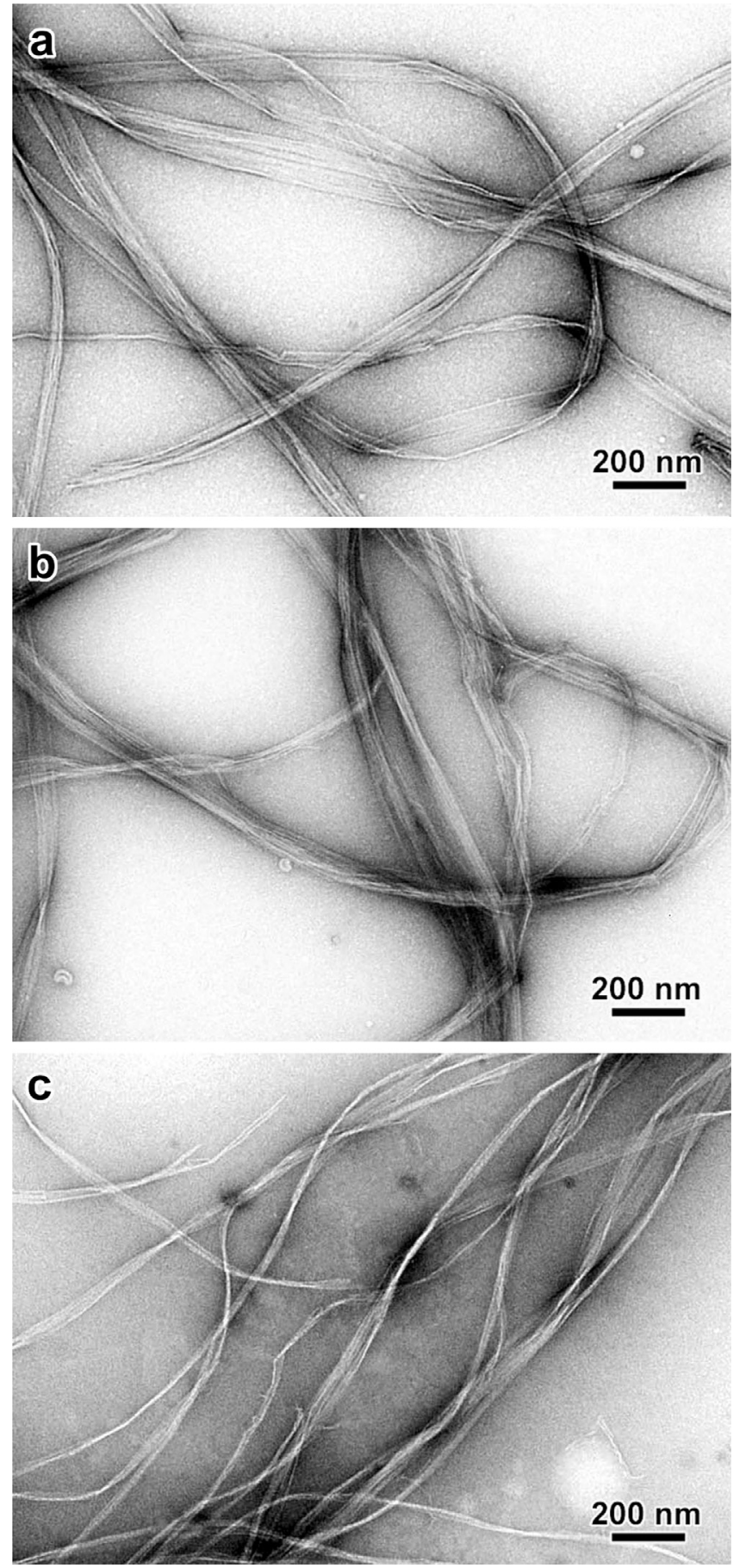

Fig. 2. TEM images of cellulose ribbons synthesized by Gluconacetobacter medellensis in unmodified media (a) glyoxal-modified media before curing (b) and glyoxal-modified media after curing (c).

The curing effect on crosslinking BC pellicle networks was evaluated by SEM. Fig. 3a shows networks of BCG cellulose ribbons after curing at $120^{\circ} \mathrm{C}$ and after purification. By comparison with $\mathrm{BC}$ and BCM (Fig. 1 a and b, respectively) a network of stretched ribbons was observed. It is important to note that this stretching state was not reversible during the purification steps, after exhaustive washing with water and treatment with $\mathrm{KOH}$. Therefore, network modification imparts stability in aqueous media. Adhesion between ribbons in the network is clearly observed in higher magnification images (Fig. 3b) and reveals the effect of the crosslinking between BCG ribbons surface in the network. 

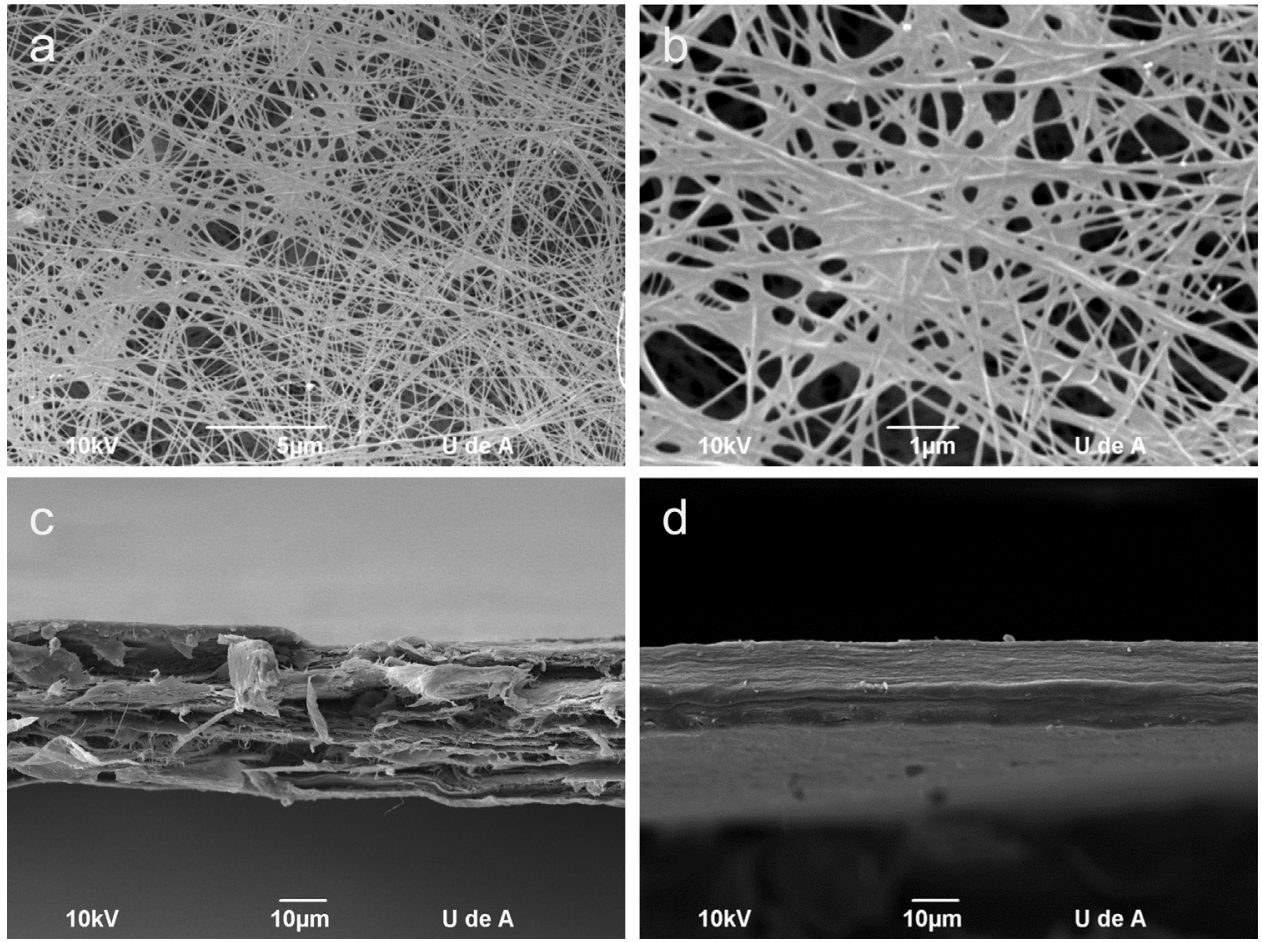

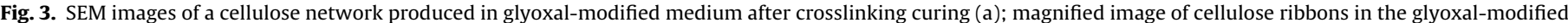
network (b); fractured section of dry bacterial cellulose film produced in unmodified (c) and in glyoxal-modified (d) media after crosslinking curing.

The fracture surfaces of dry films of BC and cross-linked BCG are shown in Fig. 3c and d, respectively. The typical delamination of BC layers is observed in the sample. However, this effect is considerably reduced when glyoxal was applied to the culture medium (BCG) and the film (produced under same conditions) was subsequently crosslinked. This is explained by the formation of covalent bonds between the cellulose ribbons, which increased interlayer adhesion.

ATR-FTIR spectra of BC and BCG (Fig. 4a) are similar, indicating the similar chemical composition of cellulose produced in the unmodified and modified media. However, the bands corresponding to acetals and hemiacetals in crosslinked cellulose from reaction between dialdehydes and cellulose cannot be distinguished in the BCG spectrum. This was explained by the fact that five-membered rings were formed after reaction between hydroxyl groups of the cellulose and glyoxal, the vibrations of which are overlapped with those of the cellulose (Schramm, \& Rinderer, 2000; Quero et al., 2011; Choi, Kim, \& Shin, 1999). Thus, FTIR was not fully suitable to confirm crosslinking reactions.

Fig. $4 \mathrm{~b}$ and $\mathrm{c}$ shows detailed ATR-FTIR spectra at $3720-2650 \mathrm{~cm}^{-1}$ and $780-400 \mathrm{~cm}^{-1}$, respectively. The absorbances at 3240 and $750 \mathrm{~cm}^{-1}$ were assigned to the triclinic $I_{\alpha}$ allomorph, and the absorbances at 3270 and $710 \mathrm{~cm}^{-1}$ were assigned to the monoclinic $I_{\beta}$ form (Sugiyama, Persson, \& Chanzy, 1991). These spectral bands were practically the same for BC and BCG, which indicated that the relative content of $I_{\alpha}$ and $I_{\beta}$ allomorphs was unaffected by the modification and cellulose synthesis in the culture medium. The addition of exogenous components into culture medium has been reported to favor $I_{\beta}$ allomorphism (thermodynamically more stable) compared to $I_{\alpha}$ (Yamamoto \& Horii, 1994; Tokoh, Takabe, Sugiyama, \& Fujita, 2002). Therefore, there is indication that glyoxal did not affect the crystallization process of cellulose in BCG.

The ATR-FTIR data are in agreement with those obtained by $\mathrm{CP} / \mathrm{MAS}{ }^{13} \mathrm{C}$ NMR (Fig. 5). BC and BCG spectra are very similar, presenting resonance lines assigned to the $\mathrm{C} 1$ (102-108 ppm), C4
(81-93 ppm) and C6 (60-70 ppm) carbons, as well as a cluster of resonances assigned to the C2, C3 and C5 carbons (Atalla, \& Vanderhart, 1984). Moreover, a slight variation in the vicinity of the cluster of carbons C2, C3 and C5 is observed in solid-state spectra. C2, C3 and C6 carbons are the atoms directly involved in glyoxal crosslinking (Schramm, \& Rinderer, 2000; Quero et al., 2011). No change was observed in the $\mathrm{C} 6$ zone, which may be explained by the fact that this signal in cellulose NMR spectra is assigned to the interactions of carbon 6 in the crystalline structure of cellulose (Lai, 1996), while in the present case the modification occurred only on the surface, i.e., the $\mathrm{OH}$ groups inside the crystal were not affected by crosslinking. A combination of the $I_{\alpha}$ and $I_{\beta}$ spectra is also seen in NMR spectra, however, the main contribution is located at $106.2 \mathrm{ppm}$, indicating that both samples are $I_{\alpha}$-rich. In order to determine possible changes in the crystallinity due to glyoxalization, the abundance of crystallites relative to amorphous cellulose was determined according to Newman (Newman, 1999) and it was found that the crystallinity remains unchanged, being $73 \%$ for both $\mathrm{BC}$ and $\mathrm{BCG}$.

Fig. $6 a$ and $b$ includes the XRD profiles of the BC and BCG samples, respectively. The diffraction patterns were indexed according to the cellulose $I_{\alpha}$ indexation described by Sugiyama et al. (Sugiyama et al., 1991). The three visible peaks were assigned to (1 000$),\left(\begin{array}{lll}0 & 1 & 0\end{array}\right)$ and $\left(\begin{array}{lll}1 & 1 & 0\end{array}\right)$ crystallographic planes, corresponding to diffraction angles of $14.4^{\circ}, 16.7^{\circ}$ and $22.7^{\circ}$, respectively. The relative intensities of the peaks reveal a strong uniplanarity in the film which can be expected considering the flat ribbon shape of the synthesized cellulose. The peaks were deconvoluted to determine the interplanar crystal distances ( $d$-spacings) and crystallite dimensions, which are listed in Table 1. Changes did not occur in the $d$-spacings of crystalline configuration of bacterial cellulose by the crosslinking (BCG) nor during cellulose synthesis in the modified culture medium, before curing (BGM). However, the crystallite was smaller in BCM and BCG compared to BC. Importantly, the values of these parameters for BCM (not shown) and BCG are the same. According to the $\mathrm{CP} / \mathrm{MAS}{ }^{13} \mathrm{C}$ NMR data, the modification and 

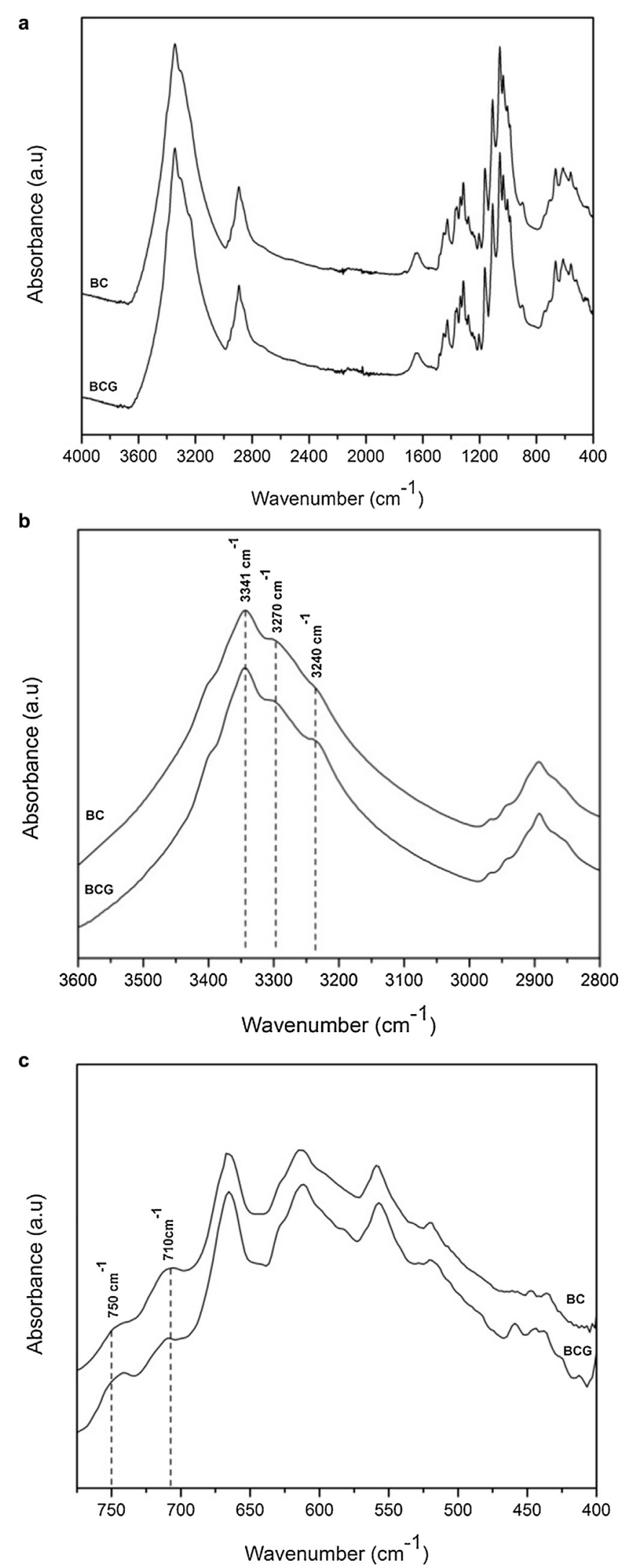

Fig. 4. ATR-FTIR spectra of unmodified and glyoxal modified bacterial cellulose (a); absorption bands at 3240 and $3271 \mathrm{~cm}^{-1}$ (b) and absorption bands at 750 and $710 \mathrm{~cm}^{-1}$ (c) correspond to $I_{\alpha}$ and $I_{\beta}$, respectively.

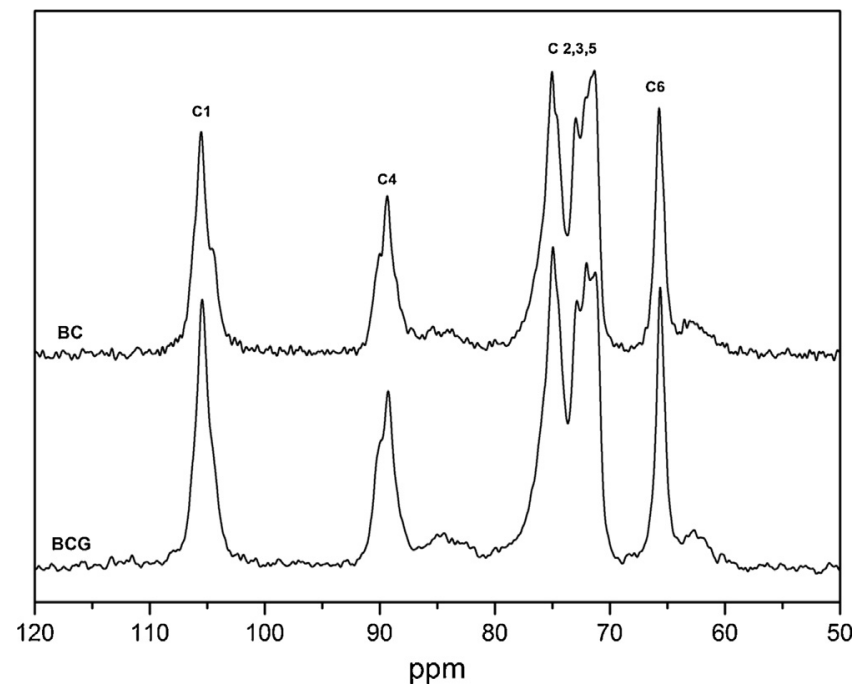

Fig. 5. ${ }^{13} \mathrm{C} \mathrm{CP} / \mathrm{MAS}$ NMR solid state spectra of unmodified and glyoxal modified bacterial cellulose.
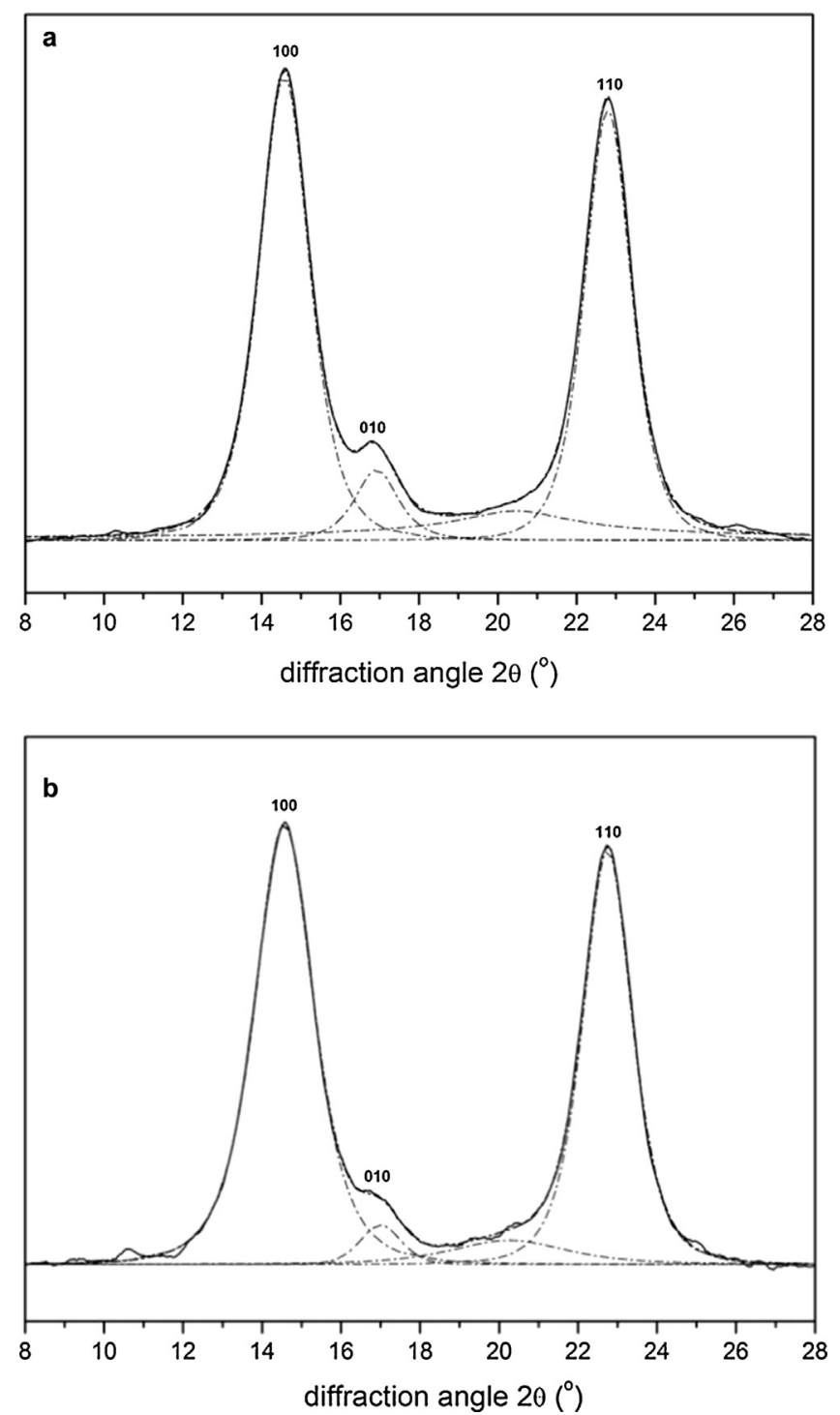

Fig. 6. X-ray diffraction spectra (reflection mode) of unmodified and glyoxalmodified bacterial cellulose. 
Table 1

$d$-Spacings $(d)$ and apparent crystallite size (ACS) in unmodified and glyoxal-modified bacterial cellulose.

\begin{tabular}{|c|c|c|c|c|c|c|}
\hline \multirow[t]{2}{*}{ Sample } & \multicolumn{2}{|l|}{$(100)$} & \multicolumn{2}{|l|}{$\left(\begin{array}{lll}0 & 1 & 0\end{array}\right)$} & \multicolumn{2}{|l|}{$(110)$} \\
\hline & $d$-Spacing $(\mathrm{nm})$ & $\mathrm{ACS}(\mathrm{nm})$ & $d$-Spacing $(\mathrm{nm})$ & ACS $(\mathrm{nm})$ & $d$-Spacing $(\mathrm{nm})$ & $\operatorname{ACS}(\mathrm{nm})$ \\
\hline $\mathrm{BC}$ & $0.609 \pm 0.0008$ & $5.294 \pm 0.0040$ & $0.525 \pm 0.0001$ & $6.627 \pm 0.2500$ & $0.390 \pm 0.0003$ & $5.811 \pm 0.0250$ \\
\hline BCG & $0.604 \pm 0.0040$ & $4.361 \pm 0.3300$ & $0.515 \pm 0.0020$ & $4.370 \pm 0.2900$ & $0.388 \pm 0.0020$ & $5.362 \pm 0.2000$ \\
\hline
\end{tabular}

Table 2

Values of dispersive component of the surface energy $\left(\gamma_{s}^{d}\right)$, acid and base constants $\left(K_{a}\right.$ and $\left.K_{b}\right)$, BET specific surface area and monolayer capacity of bacterial cellulose at $20^{\circ} \mathrm{C}$.

\begin{tabular}{lll}
\hline & \multicolumn{2}{l}{ Bacterial cellulose } \\
\cline { 2 - 3 } & Unmodified & Glyoxal-modified \\
\hline$\gamma_{s}^{d}\left(\mathrm{~mJ} \mathrm{~m}^{-2}\right)$ & 39.64 & 54.55 \\
$K_{a}$ & 0.09 & 0.11 \\
$K_{b}$ & 0.05 & 0.11 \\
$K_{a} / K_{b}$ & 1.80 & 1.00 \\
Specific surface area $\left(\mathrm{m}^{2} \mathrm{~g}^{-1}\right)$ & 1.94 & 2.97 \\
Monolayer capacity $\left(\mathrm{mmol} \mathrm{g}^{-1}\right)$ & 0.005 & 0.008 \\
\hline
\end{tabular}

the synthesis in modified medium did not alter the crystallinity of the synthesized BC. Therefore, the difference in crystallite dimensions may be associated with a decreased the number of cellulose chains forming the crystallites. Glyoxal may restrict the ability of the chains to interact through formation of hydrogen bonds to form larger crystallites during cellulose synthesis. Likewise, the crosslinking reaction occurs only on the nanofibril surfaces and hydroxyl groups located inside crystal regions that are inaccessible to chemical modification.

IGC and CA measurements were carried out to determine the changes in the surface properties of BC and BCG. The dispersive component of the surface energy, $\gamma_{s}^{d}$, of BC and BCG was obtained by plotting RTln $V_{N}$ against $a\left(\gamma_{L}^{d}\right)^{1 / 2}$ for an $n$-alkane series. The correlation coefficient obtained in this study was excellent (0.998-0.999) and the calculated values are summarized in Table 2 . The $\gamma_{s}^{d}$ found for the BC $\left(39.64 \mathrm{~mJ} \mathrm{~m}^{-2}\right)$ is in the range of those reported in the literature ( 31.7 to $49.3 \mathrm{~mJ} \mathrm{~m}^{-2}$ ) for crystalline and microcrystalline cellulose (Steele et al., 2008; Abdelmouleh, Boufi, Salah, Belgacem, 2002; Planinsek, \& Buckton, 2003). A significant increase (about $40 \%$ ) in $\gamma_{s}^{d}$ was observed upon glyoxal treatment, indicating that the glyoxalization makes the surface of bacterial cellulose more hydrophobic. It is thus expected that BCG is more compatible with hydrophobic matrices.

This behavior was also observed with the water contact angle (WCA), measurements confirmed the shift to a more hydrophobic surface after BC glyoxalization (an increase in the WCA from 65 to $75^{\circ}$ was observed).

Glyoxal is a highly polar molecule that originates polar acetal and hemiacetal structures when reacted with hydroxyl groups. However, glyoxalization increases the hydrophobicity of the cellulose polymer. This can be justified by the cellulose anisotropy. Briefly, as proposed by Yamane et al. (2006) cellulose has two inherently different domains, hydrophilic and hydrophobic. The hydroxyl groups located in equatorial positions of the glucopyranose rings are responsible for the hydrophilic behavior of cellulose. Conversely, the $\mathrm{C}-\mathrm{H}$ groups located in axial positions of the ring are responsible for its hydrophobic character. Thus, the significant increase in the hydrophobic character of the $\mathrm{BC}$ suggests that glyoxalization affects the structural organization of the glucopyranose ring orientation. Therefore, as a result of the reaction between the cellulose hydroxyl groups with the dialdehyde function of the glyoxal, the parallel cellulose chains are oriented so that the $\mathrm{C}-\mathrm{H}$ bonds are exposed and become more accessible on the surface of the cellulose chain. This organization of the non-polar groups is reflected in the displacement of the peak maximum of the heterogeneity profile (Fig. 7a) towards higher energies (7.90 to $10.07 \mathrm{~kJ} \mathrm{~mol}^{-1}$ ), which indicate that the hydrophobicity increased due to the energy increase of the non-polar active sites after glyoxal modification.

The cellulose chains contain polar groups that are able to exchange specific interactions with polar probes and could be studied by the specific component of surface free energy, $\Delta G_{\text {ads }}^{\text {sp }}$. Thus, $\Delta G_{\text {ads }}^{\text {sp }}$ was determined by IGC by applying five polar probes: acetonitrile, acetone, ethanol, tetrahydrofuran and ethyl acetate. The obtained $\Delta G_{\mathrm{s}}^{\mathrm{sp}}$ values are included in Table 3 . The acetonitrile probe (amphoteric probe) displays a stronger interaction with the celluloses indicating acidic and basic character on the surfaces. A general increase in $\Delta G_{\mathrm{s}}^{\mathrm{sp}}$ for all the probes, was observed after glyoxalization. This indicates an increase of the polar groups in the BCG surface. The energy profile obtained from the acid probe (ethanol) and from the basic probe (tetrahydrofuran), supports this observation, as shown in Fig. 7b. The peak maximum increases for both probes, and the high energy sites display a larger population $(\mathrm{d} N / \mathrm{d} A)$, indicating an increase in the specific interaction of the BCG surface. This could be a result of new polar groups $(\mathrm{OH}$ and $\mathrm{C}-\mathrm{O}-\mathrm{C}$ ) from the hemiacetal and acetal structures formed by cellulose glyoxalization.

The obtained values of $\Delta G_{\text {ads }}^{\mathrm{sp}}$ were converted into acid-base numbers using the Gutmann's concept (Gutmann, 1978). The linear fit ranged from 0.994 and 0.995 , which means that the Gutmman's acid-concept is valid for the samples under study and the specific interactions may be considered as being of the electron donor-acceptor type. Table 2 shows the values obtained for acid-base constants $\left(K_{a}\right.$ and $\left.K_{b}\right)$. Crystalline and microcrystalline cellulose present $K_{a} / K_{b}$ ratios between 0.36 and 1.82 (Abdelmouleh et al., 2002; Papirer, Brendle, Balard, \& Vergelati, 2000). In our study, a $K_{a} / K_{b}$ ratio of 1.80 was obtained for BC. After glyoxal treatment an increase in both constants was observed, however a higher $K_{b}$ increase was detected. This could be due to the higher inclusion of basic groups $(\mathrm{C}-\mathrm{O}-\mathrm{C})$ from hemiacetal and acetal structures compared to the acid groups $(\mathrm{OH})$. These new acid and basic groups explain that at the surface of BCG presents a similar acid-basic character $\left(K_{a} / K_{b}=1\right)$. Quero et al. also found an increase in the acid groups on the surface of BC after glyoxalization (Quero et al., 2011).

Table 3

Specific free energy of adsorption $\left(\Delta G_{\mathrm{ads}}^{\mathrm{sp}} ; \mathrm{kJ} \mathrm{mol}^{-1}\right)$ obtained for unmodified and glyoxal-modified bacterial cellulose at $20{ }^{\circ} \mathrm{C}$.

\begin{tabular}{|c|c|c|c|c|c|}
\hline \multirow[t]{2}{*}{ Sample } & \multicolumn{5}{|c|}{ Specific free energy of adsorption $\left(\Delta G_{\mathrm{ads}}^{\mathrm{sp}} ; \mathrm{kJ} \mathrm{mol}^{-1}\right)$} \\
\hline & Tetrahydrofuran & Ethanol & Acetone & Ethyl acetate & Acetonitrile \\
\hline $\mathrm{BC}$ & 8.004 & 7.155 & 5.781 & 7.216 & 9.493 \\
\hline BCG & 9.378 & 9.802 & 7.439 & 8.865 & 11.81 \\
\hline
\end{tabular}



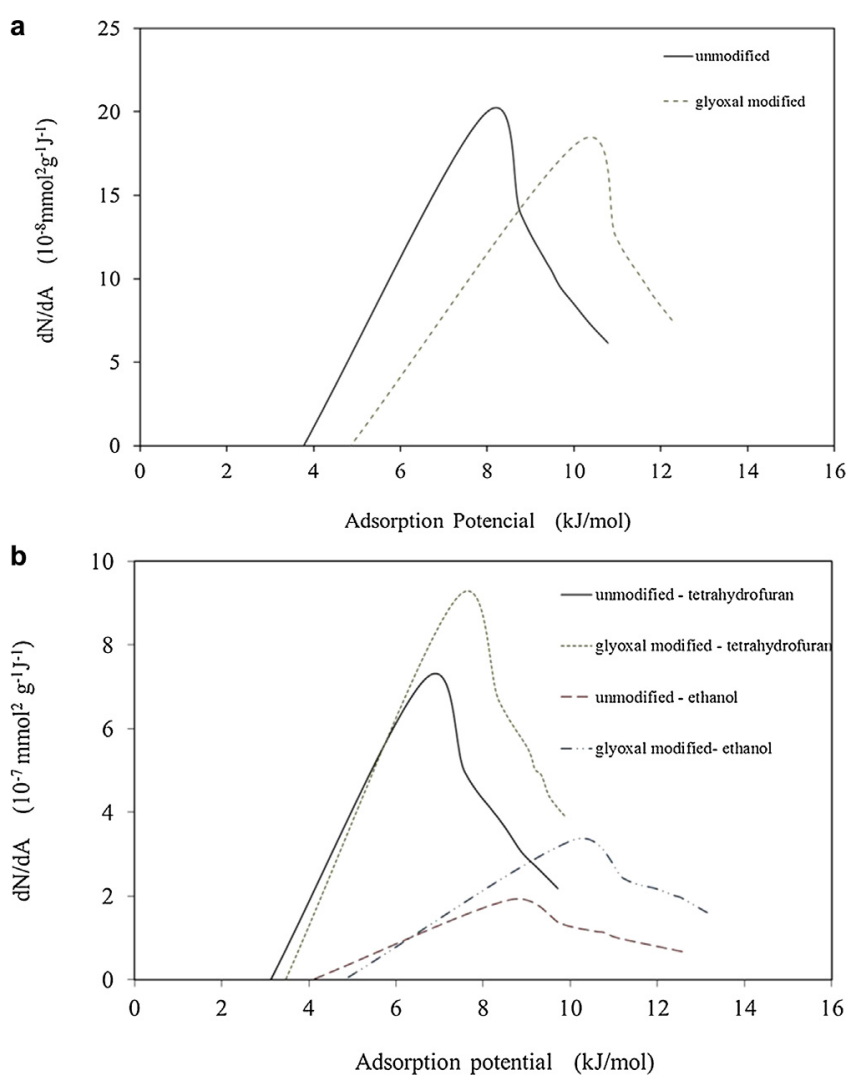

Fig. 7. Energetic profiles obtained with $n$-octane (a), ethanol and tetrahydrofuran (b), for unmodified and glyoxal-modified bacterial cellulose, at $20^{\circ} \mathrm{C}$.

In summary, the results indicate that the changes in the surface of bacterial cellulose are due to the formation of covalent bonds between the hydroxyl groups of cellulose ribbons and glyoxal, which produces changes in the orientation of the glucopyranose rings. Considering ATR-FTIR and $\mathrm{CP} / \mathrm{MAS}{ }^{13} \mathrm{C}$ NMR observations and also the fact that IGC at infinite dilution is surface-sensitive, this indicates that the modification carried out with relatively small amounts of glyoxal modified the surface of BC. That is, the reaction between the hydroxyl groups of the cellulose and the glyoxal only occurred on the surface, and thus the crystalline structure of cellulose was preserved. This observation is in agreement with that of Quero et al. (2011) after glyoxalization of sheets of bacterial cellulose.

IGC also shows significant changes in the morphology of the bacterial cellulose after glyoxal modification. Table 2 includes the values of the surface area and monolayer capacity obtained by fitting BET isotherms after measurements with $n$-octane. An increase in the surface area was observed in BCG due to the decrease in ribbons width and their stretching by curing, as observed by TEM and SEM. The higher surface area presented by the bacterial cellulose formed during glyoxalization induced an increase in the monolayer capacity (Table 2), which is expected to improve the surface interactions and support the increase in the surface energy.

\section{Conclusions}

Glyoxal, a non-formaldehyde crosslinking agent, was added in the culture medium of Gluconacetobacter bacteria. Crosslinking reactions induced by thermal curing after synthesis caused a stretching in the ribbons in the network and their fusion. This observation indicates the establishment of covalent bonds between glyoxal and the surfaces of the ribbons. Evidence of this type of linkage could not be followed by FTIR. However, the change of the surface from acidic to amphoteric suggests that the reaction between hydroxyl groups of the cellulose and the glyoxal occurred only at the surface. Despite the fact that the ribbons were biosynthesized in a glyoxal-supplemented medium, no change in the crystallinity of cellulose was observed, which further indicates that the modification only occurred on the surface and did not involve $\mathrm{OH}$ groups in the crystals. An increase of $10^{\circ}$ in contact angle also indicates an increased hydrophobicity of glyoxalizated BC films, in agreement with IGC experiments, which reveals an increase in the dispersive component of the surface energy.

\section{Acknowledgements}

The authors acknowledge the "Programa Nacional de Reequipamento Científico", POCI 2010, for sponsoring the IGC work (FEDER and Foundation for the Science and Technology), and to the Colciencias and SENA for financial support. Orlando Rojas is grateful for funding support by the Academy of Finland through its Centers of Excellence Program (2014-2019), under project "Molecular Engineering of Biosynthetic Hybrid Materials Research" (HYBER) and the project "Nanocellulose Assemblies for Microsensing and Fluidics" of TEKES (Finland).

\section{References}

Abdelmouleh, M., Boufi, S., Salah, A., \& Belgacem, M. N. (2002). Interaction of silane coupling agents with cellulose. Langmuir, 18, 3203-3208.

Atalla, R. H., \& Vanderhart, D. L. (1984). Native cellulose: A composite of two distinct crystalline forms. Science, 223, 283-285.

Bielecki, S., Krystynowicz, A., Turkiewicz, M., \& Kalinowska, H. (2005). Bacteria cellulose. In A. Steinbüchel, \& Y. Doi (Eds.), Biotechnology of biopolymers: From synthesis to patents (pp. 381-434). Weinheim, Germany: Wiley-VCH.

Castro, C., Zuluaga, R., Cleenwerck, I., Trcek, J., De Vos, P., Putaux, J.-L., et al. (2013) Gluconacetobacter medellensis sp. nov., a novel cellulose-producing bacterium isolated from homemade fruit vinegar and reclassified species of Gluconacetobacter xylinum. International Journal of Systematic and Evolutionary Microbiology, 63, 1119-1125.

Choi, H-M., Kim, J. H., \& Shin, S. (1999). Characterization of cotton fabrics treated with glyoxal and glutaraldehyde. Journal of Applied Polymer Science, 73, 2691-2699.

Cordeiro, N., Gouveia, C., Moraes, A. G. O., \& Amico, S. C. (2011). Natural fibers characterization by inverse gas chromatography. Carbohydrate Polymers, 84, 110-117.

Gutmann, V. (1978). The donor-acceptor approach to molecular interactions. New York, NY: Plenum Publ. Corp.

Head, F. S. H. (1958). The reactions of cellulose with glyoxal. Journal of the Textile Institute Transactions, 49, 345-356.

Heinze, T., \& Liebert, T. (2001). Unconventional methods in cellulose functionalization. Progress in Polymer Science, 26, 1689-1762.

Iguchi, M., Yamanaka, S., \& Budhiono, A. (2000). Review: Bacterial cellulose-A masterpiece of nature's arts. Journal of Materials Science, 35, 261-270.

Kielhorn, J., Pohlenz-Michel, C., Schmidt, S., \& Mangelsdorf, I. (2004). Glyoxal. In World Health Organization (concise international chemical assessment document 57). World Health Organization: Geneva.

Laborie, M. P. (2009). Bacterial cellulose and its polymeric nanocomposites. In L. Lucia, \& O. Rojas (Eds.), The nanoscience and technology of renewable biomaterials (pp. 231-271). Chichester, UK: John Wiley \& Sons, Ltd.

Lai, Y. Z. (1996). Reactivity and accessibility of cellulose, hemicellulose, and lignins. In D. N-S. Hon (Ed.), Chemical modification of lignocellulosic materials (pp. 35-95). New York, NY: Marcel Dekker, Inc.

Lee, E. S., \& Kim, S. I. (2005). Effect of additives on durable-press cotton fabrics treated with a glyoxal/glycol mixture. Journal of Applied Polymer Science, 96, 975-978.

Newman, R. (1999). Estimation of the lateral dimensions of cellulose crystallites using ${ }^{13} \mathrm{C}$ NMR signal strengths. Solid State Nuclear Magnetic Resonance, 15, 21-29.

Papirer, E., Brendle, E., Balard, H., \& Vergelati, C. (2000). Inverse gas chromatography investigation of the surface properties of cellulose. Journal of adhesion science and technology, 14, 321-337.

Planinsek, O., \& Buckton, G. J. (2003). Inverse gas chromatography: Considerations about appropriate use for amorphous and crystalline powders. Journal of Pharmaceutical Sciences, 92, 1286-1294.

Quero, F., Nogi, M., Lee, K-Y., Poel, G. V., Bismarck, A., Mantalaris, A., et al. (2011). Cross-linked bacterial cellulose networks using glyoxalization. ACS Applied Materials \& Interfaces, 3, 490-499.

Schramm, C., \& Rinderer, B. (2000). Determination of cotton-bound glyoxal via an internal cannizzaro reaction by means of high-performance liquid chromatography. Analytical Chemistry, 72, 5829-5833.

Schramm, C., \& Rinderer, B. (2002). Nonformaldehyde durable press finishing of cotton fabric: Quantitative evaluation of cellulose-bound glyoxal. Textile Research Journal, 72, 357-360. 
Steele, D. F., Moreton, R. C., Staniforth, J. N., Young, P. M., Tobyn, J., \& Edge, S. (2008) Surface energy of microcrystalline cellulose determined by capillary intrusion and inverse gas chromatography. Journal of the American Association of Pharmaceutical Scientists, 10, 494-503.

Sugiyama, J., Persson, J., \& Chanzy, H. (1991). Combined infrared and electron diffraction study of the polymorphism of native cellulose. Macromolecules, 24 2461-2466.

Tokoh, C., Takabe, K., Sugiyama, J., \& Fujita, M. (2002). Cellulose synthesized by Acetobacter xylinum in the presence of plant cell wall polysaccharides. Cellulose, 9 65-74.

Welch, C. (1983). Glyoxal as a formaldehyde-free durable press reagent for mild curing applications. Textile Research Journal, 53, 181-186.
Xu, G. G., Yang, C. Q., \& Deng, Y. (2001). Applications of bifunctional aldehydes to improve paper wet strength. Journal of Applied Polymer Science, 83, 2539-2547.

Yamamoto, H., \& Horii, F. (1994). In situ crystallization of bacterial cellulose I. Influences of polymeric additives, stirring and temperature on the formation cellulose $\mathrm{I} \alpha$ and $\mathrm{I} \beta$ as revealed by cross polarization/magic angle spinning (CP/MAS) ${ }^{13} \mathrm{C}$ NMR spectroscopy. Cellulose, 1,57-66.

Yamane, C., Aoyagi, T., Ago, M., Sato, K., Okajima, K., \& Takahashi, T. (2006). Two different surface properties of regenerated cellulose due to structural anisotropy. Polymer Journal, 38, 819-826.

Yu, Y., Lee, E., \& Bang, E. (2008). A new catalyst for glyoxal durable press finish of cotton fabrics. Fibers and Polymers, 9, 715-719. 